\title{
Evaluation of a Handheld Dermatoscope in Clinical Diagnosis of Primary Cicatricial Alopecias
}

\author{
Özlem Karadag Köse · A. Tülin Güleç
}

Received: April 8, 2019 / Published online: June 12, 2019

(C) The Author(s) 2019 particular types of primary cicatricial alopecias: "target" pattern blue-grey dots, perifollicular scaling, perifollicular cast in lichen planopilaris $(n=27)$; short vellus hairs, tufted hairs, crust formation, yellowish tubular scaling, pustule, red dots in folliculitis decalvans $(n=17)$; large keratotic yellow dots in discoid lupus erythematosus $(n=7)$; yellow dots, yellow dots with "three-dimensional" structure, black dots in dissecting cellulitis of the scalp $(n=6)$. Absence of vellus hairs was observed in patients with lichen planopilaris, frontal fibrosing alopecia, and discoid lupus erythematosus without a significant difference between the groups. Short vellus hairs were detected in all types, including frontal fibrosing alopecia $(n=7)$.

Conclusion: We suggest that a polarized-light handheld dermatoscope is useful for revealing several typical trichoscopic features of primary cicatricial alopecias that guide clinical diagnosis. As a novel observation, our data indicate that absence of vellus hairs is not an identifying feature for frontal fibrosing alopecia.

Keywords: Alopecia; Dermoscopy; Hair; Handheld dermatoscope; Primary cicatricial alopecia; Trichoscopy

\section{INTRODUCTION}

Primary cicatricial alopecias (PCAs) are a rare group of disorders characterized by destruction 
of hair follicles, leading to irreversible hair loss. Detailed clinical evaluation along with careful history-taking is frequently sufficient to distinguish between PCAs and noncicatricial alopecias. However, histopathologic confirmation is needed for accurate diagnosis and identification of the different types of PCA. Nevertheless, histopathologic features are not always diagnostic [1]. Therefore, trichoscopy has become a complementary useful tool to achieve a better clinicopathologic correlation, leading to precise diagnosis. However, few comprehensive studies evaluating trichoscopic findings of PCAs are available [2, 3]. Most other relevant work has focused on either a particular disease such as frontal fibrosing alopecia (FFA) [4, 5] or dissecting cellulitis of the scalp (DCS) $[6,7]$, or included a limited number of patients [6, 8-14].

This study was undertaken to evaluate the utility of a handheld dermatoscope in clinical diagnosis of PCAs.

\section{METHODS}

\section{Study Population}

This prospective study included 69 consecutive patients with a diagnosis of PCA (29 female, 40 male) who attended the Dermatology Department of Başkent University in Ankara, Turkey, between July 2010 and December 2014. The mean age of the patients was 40.7 years (range 15-85 years). Patients with a diagnosis of seborrheic dermatitis, psoriasis, or secondary cicatricial alopecia were excluded from the study.

The study was approved by Başkent University Institutional Review Board and Ethics Committee (project no. K17/171), and the study was conducted in accordance with the principles of the Declaration of Helsinki of 1964 and its later amendments. All participants gave informed consent before study participation.

\section{Study Procedure}

Dermatologic evaluation included clinical observation along with trichoscopy. Trichoscopic examination was performed by polarized-light handheld dermatoscope (Dermlite II
Pro HR, 3Gen LLC, San Juan Capistrano, CA), which permits scalp visualization at tenfold magnification, without an interface solution as dry dermoscopy is essential to recognize scaling. Images were captured by a digital camera (Sony Cyber-shot DSC-W70, Sony Corp, Tokyo, Japan) that produced imagery with 30 -fold magnification via a $3 \times$ optical zoom lens. In each case, several different images were obtained at 10 - to 30 -fold magnifications, and evaluated in accordance with the checklist of trichoscopic features presented in Table 1.

Two trichoscopy-guided 4-mm scalp biopsy specimens were taken for vertical and horizontal sectioning, and final diagnosis was confirmed by histopathologic examination in all cases.

\section{Statistical Analysis}

Continuous variables are presented as mean \pm standard deviation (SD), and discrete variables as percentages. The Pearson chi-square test was used for intergroup comparisons, with $P$ values less than 0.05 considered significant. SPSS version 22.0 statistical software package for Microsoft Windows (SPSS Inc., Chicago, IL) was used throughout.

\section{RESULTS}

\section{Trichoscopic Features}

The number of patients with each type of PCA, and the distribution and prevalence rates of the trichoscopic features, are presented in Table 2.

\section{Follicular Features}

\section{Follicular Openings}

Absence of follicular openings (Fig. 3c) was noted in every patient with PCA. There were three types of yellow dots, namely yellow dots (Fig. 2e), yellow dots with "three-dimensional (3D)" structure (Fig. 5b, c), and large keratotic yellow dots (Fig. 4a). Yellow dots were significantly more common in DCS than the others, whereas yellow dots with "3D" structure and 
Table 1 Evaluation checklist of trichoscopic features of primary cicatricial alopecias in this study

\begin{tabular}{ll}
\hline Follicular features & Follicular and interfollicular features \\
\hline Follicular openings & Pink-white appearance \\
Absence of follicular openings & Yellow structureless areas \\
Yellow dots & Hyperpigmentation pattern \\
Yellow dots & Honeycomb pattern \\
"3D"-structured yellow dots & Brown scattered pattern \\
Large keratotic yellow dots & Crust formation \\
Black dots & Scaling \\
White dots & Interfollicular scaling \\
Fibrotic white dots & Perifollicular scaling \\
Pinpoint white dots & Peripilar cast \\
Blue-grey dots & Yellowish tubular scaling \\
"Target" pattern & Pustule \\
"Speckled" pattern & Blood vessels \\
Follicular red dots & Red dots \\
Perifollicular erythema & Arborizing red lines \\
Hair shafts & Elongated vascular loops \\
Absence of vellus hairs & Pinpoint vessels with whitish halo \\
Short vellus hairs & \\
Regrowing hairs & \\
Broken hairs & \\
Tufted hairs & \\
Pili torti & \\
& \\
\hline
\end{tabular}

large keratotic yellow dots were noted only in DCS and discoid lupus erythematosus (DLE), respectively. Black dots (Fig. 5a, b) were significantly associated with DCS. There was no significant difference between PCAs regarding fibrotic, or pinpoint white dots (Fig. 5a). "Target" pattern blue-grey dots (Fig. 1b) were found only in lichen planopilaris (LPP), while "speckled" pattern blue-grey dots, follicular red dots, and perifollicular erythema were not observed in any of the patients.

\section{Hair Shafts}

Absence of vellus hairs was observed in some patients with LPP, FFA, and DLE without a significant difference between the groups. The prevalence of short vellus hairs (Fig. $3 \mathrm{a}-\mathrm{c}$ ) was significantly higher in folliculitis decalvans (FD), as compared with the others. Regrowing hairs were only seen in FD (Fig. 2e). Broken hairs (Figs. 2c, 5a, b) were found in all types except pseudopelade of Brocq (PB), without a significant difference in frequency. Tufted hairs (Figs. 1d, 5c) were significantly prominent in FD 
Table 2 Trichoscopic features observed by handheld dermatoscope in patients with primary cicatricial alopecias

\section{Trichoscopic features}

\begin{tabular}{lllllll}
\multicolumn{4}{l}{ Type of primary cicatricial alopecia } & & & \\
\hline LPP, & FD, & FFA, & DLE, & DCS, & PB, & P values \\
$n=27(\%)$ & $n=17(\%)$ & $n=7(\%)$ & $n=7(\%)$ & $n=6(\%)$ & $n=5(\%)$ &
\end{tabular}

\section{Follicular features}

\section{Follicular openings}

Absence of follicular openings $27(100) \quad 17(100) \quad 7(100) \quad 7(100) \quad 6(100) \quad 5(100) \quad-$

Yellow dots

Yellow dots

$1(3.7)$

$4(23.5)$

$2(28.6)$

$-$

$5(83.3)$

$<0.001$

“3D”-structured yellow dots

$-$

Large keratotic yellow dots

Black dots

$4(14.8)$

$-$

White dots

Fibrotic white dots

$11(40.7)$

$2(11.8)$

$3(42.9)$

$2(28.6)$

$6(100)$

$<0.001$

Pinpoint white dots

$4(14.8)$

1 (14.3)

$3(42.9)$

$<0.001$

“Target” pattern blue-grey dots 7 (25.9)

$-$

$-$

$1(16.7)$

$1(20)$

0.172

Hair shafts

Absence of vellus hairs

8 (29.6) -

$2(28.6)$

2 (28.6)

$-$

0.077

Short vellus hairs

$1(3.7)$

$8(47.1)$

3 (42.9)

1 (14.3)

1 (16.7)

$-$

0.017

Regrowing hairs

- 2 (11.8)

$-$

9 (33.3)

7 (41.2)

1 (14.3)

-

Broken hairs

5 (18.5)

$14(82.4)$

-

14 (51.9)

$8(47.1)$

$5(71.4)$

$1(14.3)$

$-$

0.278

Tufted hairs

Pili torti

\section{features}

Pink-white appearance

$27(100)$

$17(100)$

$7(100)$

7 (100)

$6(100)$

$5(100)$

Yellow structureless areas

-

Hyperpigmentation pattern

Honeycomb pattern

$15(55.6)$

$3(17.6)$

$5(71.4)$

1 (14.3)

$3(50)$

$2(40)$

0.051

Brown scattered pattern

$3(11.1)$

1 (5.9)

1 (14.3)

2 (28.6)

$-$

0.501

Crust formation

$4(14.8) \quad 12(70.6)$

1 (14.3)

$2(33.3)$

$<0.001$

Scaling

Interfollicular scaling

$15(55.6)$

$3(17.6)$

$3(42.9)$

6 (85.7)

2 (33.3)

0.084

Perifollicular scaling

22 (81.5)

$8(47.1)$

$5(71.4)$

$3(42.9)$

1 (16.7)

1 (20)

$<0.001$

Peripilar cast

$14(51.9)$

$5(29.4)$

1 (14.3)

1 (14.3)

1 (20)

0.013

Yellowish tubular scaling

$-$

$12(70.6)$

1 (16.7)

$<0.001$

Pustule

$1(3.7)$

15 (88.2)

1 (16.7)

$<0.001$ 
Table 2 continued

\begin{tabular}{|c|c|c|c|c|c|c|c|}
\hline \multirow[t]{2}{*}{ Trichoscopic features } & \multicolumn{7}{|c|}{ Type of primary cicatricial alopecia } \\
\hline & $\begin{array}{l}\text { LPP, } \\
n=27 \text { (\%) }\end{array}$ & $\begin{array}{l}\text { FD, } \\
n=17 \text { (\%) }\end{array}$ & $\begin{array}{l}\text { FFA, } \\
n=7 \text { (\%) }\end{array}$ & $\begin{array}{l}\text { DLE, } \\
n=7(\%)\end{array}$ & $\begin{array}{l}\text { DCS, } \\
n=6(\%)\end{array}$ & $\begin{array}{l}\mathrm{PB}, \\
n=5(\%)\end{array}$ & $P$ values \\
\hline \multicolumn{8}{|l|}{ Blood vessels } \\
\hline Red dots & $8(29.6)$ & $16(94.1)$ & $1(14.3)$ & $2(28.6)$ & $3(50)$ & $1(20)$ & $<0.001$ \\
\hline Arborizing red lines & $10(37)$ & $11(64.7)$ & $3(42.9)$ & $3(42.9)$ & $2(33.3)$ & $4(80)$ & 0.314 \\
\hline
\end{tabular}

$D C S$ dissecting cellulitis of the scalp, $D L E$ discoid lupus erythematosus, $F D$ folliculitis decalvans. FFA frontal fibrosing alopecia, $L P P$ lichen planopilaris, $P B$ pseudopelade of Brocq

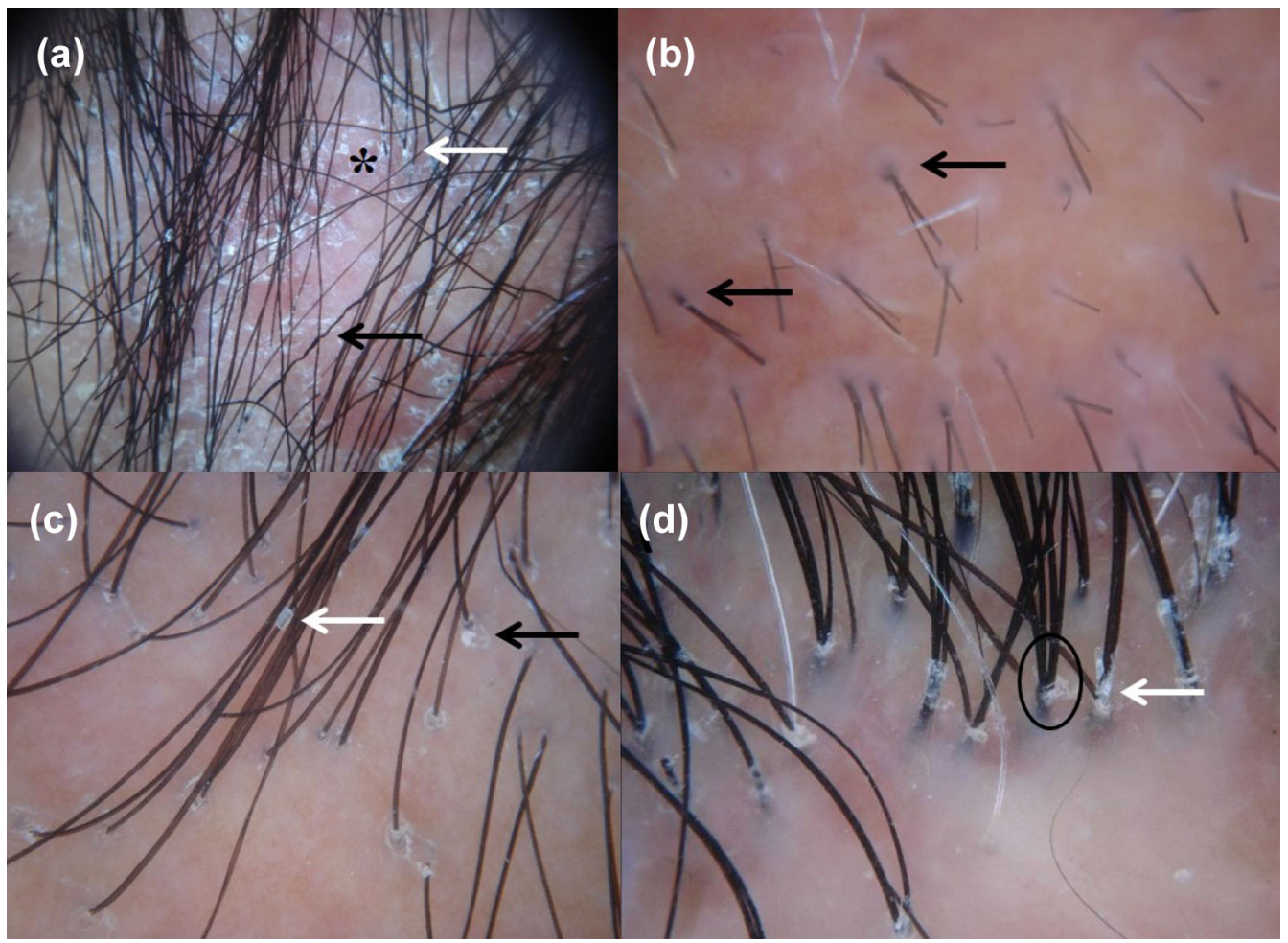

Fig. 1 Trichoscopic features of scalp in lichen planopilaris. a Interfollicular scaling (asterisk), perifollicular scaling (white arrow), and pili torti (black arrow). b "Target" pattern blue-grey dots (black arrows). c Peripilar cast

(Fig. 2d). There was no significant difference between PCAs regarding pili torti (Figs. 1a, 3b).

\section{Follicular and Interfollicular Features}

Pink-white appearance (Figs. 2c, 5c) was detected in all subjects with PCA, while yellow (white arrow) and perifollicular scaling (black arrow). d Tufted hairs (circle) and perifollicular scaling (white arrow) (original magnifications: $\mathbf{a} \times 10 ; \mathbf{b}-\mathbf{d} \times 30$ )

structureless areas were observed solely in DCS. There was no significant difference between PCAs regarding honeycomb pigment pattern (Fig. 3a) or brown scattered pattern (Fig. 4a, b). Crust formation (Fig. 2a) was significantly more common in FD than the others. Interfollicular scaling (Figs. 1a, 2d, 4c) was encountered in all types except $\mathrm{PB}$, without a significant difference 


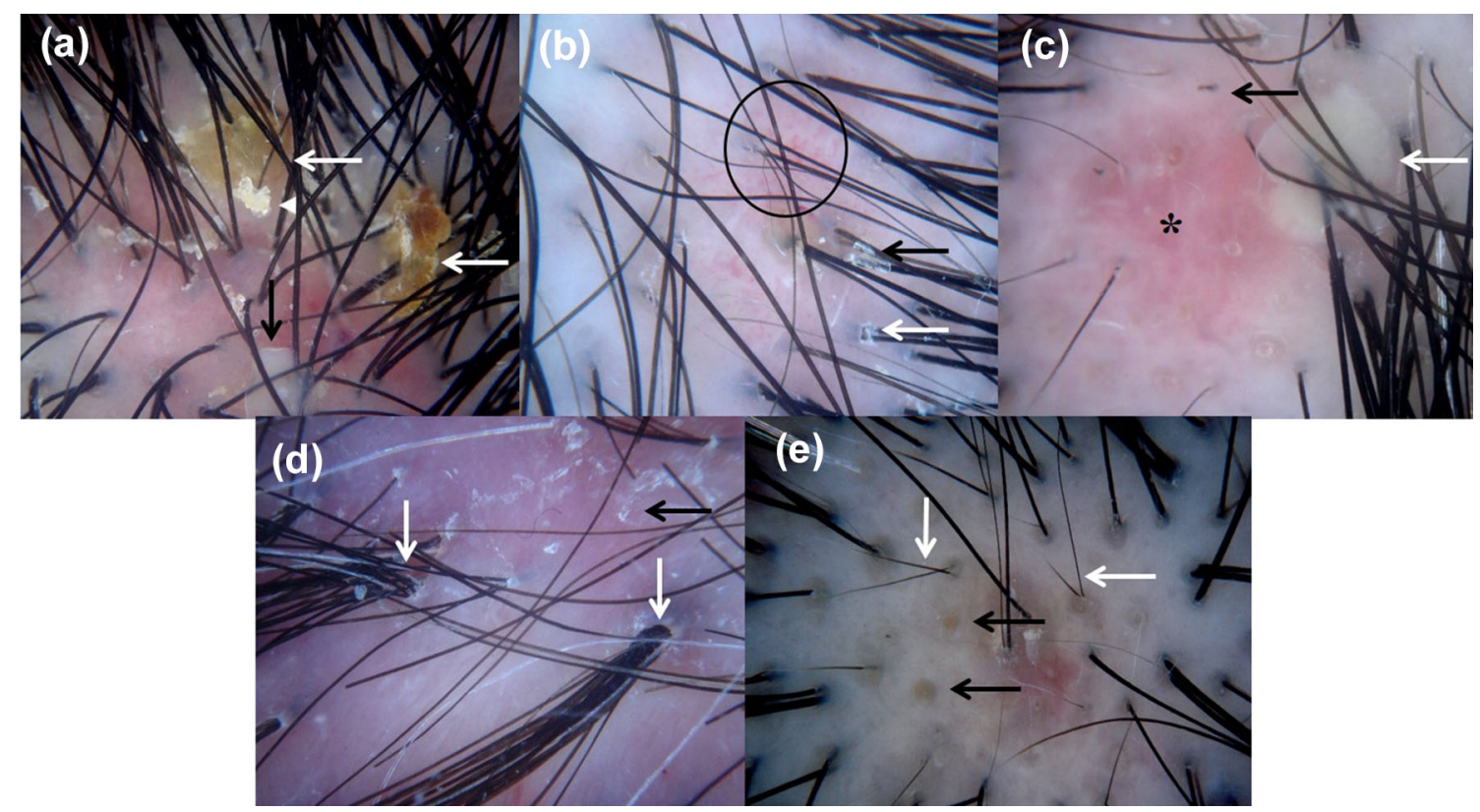

Fig. 2 Trichoscopic features of scalp in folliculitis decalvans. a Crust formation (white arrows), yellowish tubular scaling (white arrowhead), and pustule (black arrow). b Red dots (circle), peripilar cast (black arrow), and perifollicular scaling (white arrow). c Broken hair (black arrow), pustule (white arrow), and pink-white appearance (asterisk). d Interfollicular scaling (black arrow) and tufted hairs (white arrows). e Regrowing hairs (white arrows) and yellow dots (black arrows) (original magnifications: a-e $\times 30$ )

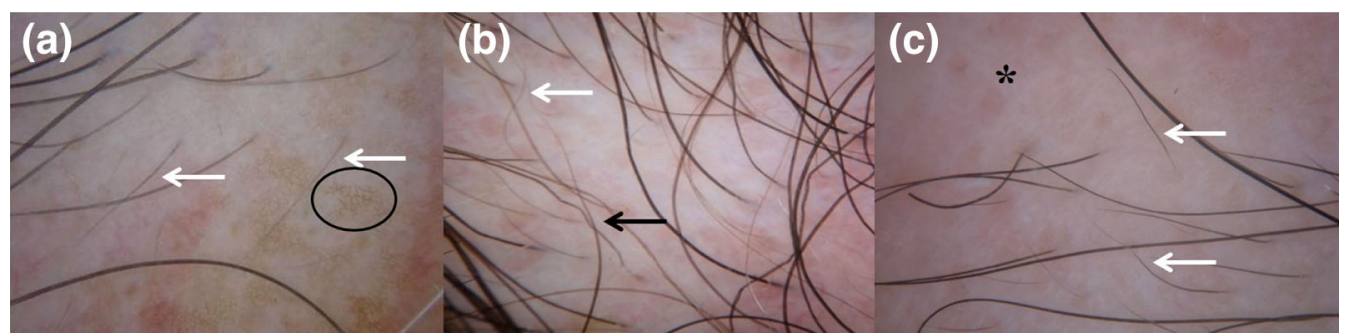

Fig. 3 Trichoscopic features of scalp in frontal fibrosing alopecias. a Short vellus hairs (white arrows) and honeycomb pigment pattern (circle). b Short vellus hair (white

in frequency. Perifollicular scaling (Figs. 2b, 4b, c) and peripilar casts (Fig. 2b) were significantly associated with LPP (Fig. 1a, c, d), whereas yellowish tubular scaling (Fig. 2a) and pustules (Fig. 2a, c) were significantly prominent in FD.

\section{Blood Vessels}

Red dots (Fig. 5c) were significantly associated with FD (Fig. 2b), while no significant difference between PCAs concerning arborizing red lines (Fig. 4b) was noted. Elongated vascular loops arrow) and pili torti (black arrow). c Absence of follicular openings (asterisk) and short vellus hairs (white arrows) (original magnifications: $\mathbf{a}-\mathbf{c} \times 30$ )

and pinpoint vessels with whitish halo were not observed in any of the participants.

Red dots could only be appreciated at 30-fold magnification; the rest of the trichoscopic features were easily viewed at magnifications of 10to 30 -fold.

\section{DISCUSSION}

Our previous study [8], which was the first to investigate PCAs using a handheld 


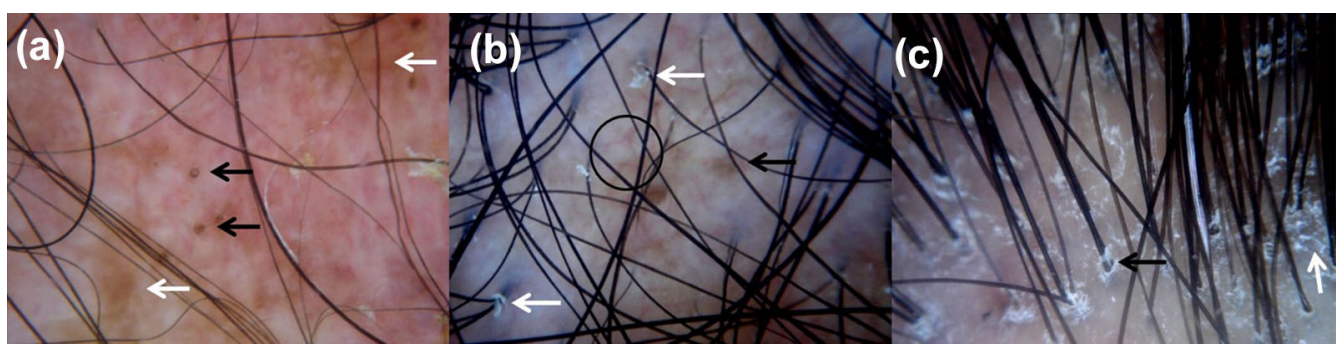

Fig. 4 Trichoscopic features of scalp in discoid lupus erythematosus. a Brown scattered pigmentation (white arrows) and keratotic yellow dots (black arrows). b Perifollicular scaling (white arrows), arborizing red lines (circle), and brown scattered pigmentation (black arrow). c Perifollicular scaling (black arrow) and interfollicular scaling (white arrow) (original magnifications: $\mathbf{a}-\mathbf{c} \times 30$ )

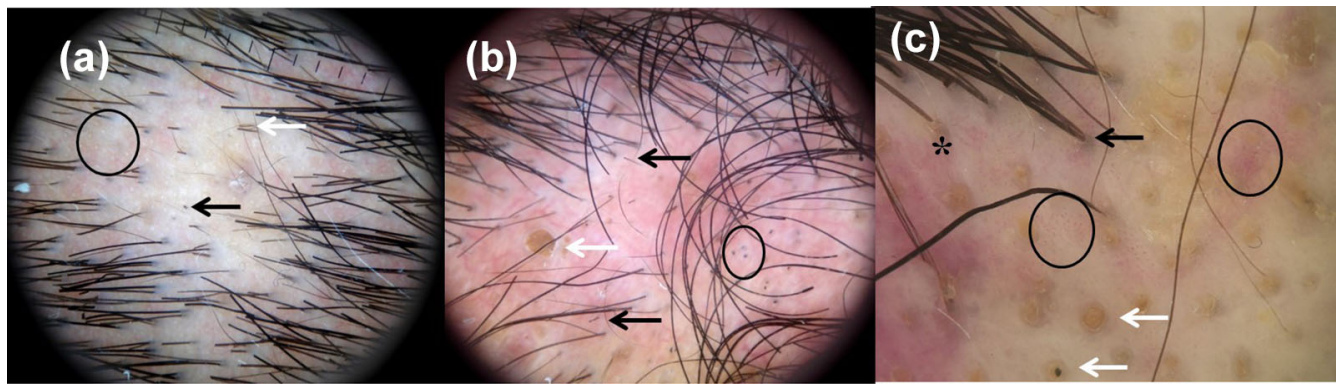

Fig. 5 Trichoscopic features of scalp in dissecting cellulitis. a Pinpoint white dots (circle), broken hairs (white arrow), and black dot (black arrow). b Broken hairs (black arrows), yellow dot with “3D” structure (white arrow), and

dermatoscope, included only 21 patients with PCA among 144 participants with a diagnosis of alopecia. Therefore, it carried the limitation of evaluating the whole PCA group in the same cluster due to the small sample size. Absence of follicular openings was observed in all subjects [8] and considered as a hallmark feature for PCAs, confirming a prior study [11]. Likewise, tufted hairs, follicular hyperkeratosis, pili torti, and pink-white appearance were accepted as other specific trichoscopic patterns of PCAs [8]. In 2012, another study about the trichoscopic findings of PCAs using a videodermoscope was published by Rakowska et al. [2]. They reported that white and milky-red areas lacking follicular openings were found in all types of PCA, except DCS. Recently, Abedini et al. [3] assessed the validity of trichoscopy in 100 patients with PCA using a handheld dermatoscope. They declared the absence of follicular openings, perifollicular scale, and the presence of one scarring pattern black dots (circle). c Tufted hairs (black arrow), red dots (circles), pink-white appearance (asterisk), and yellow dots with “3D” structure (white arrows) (original magnifications: $\mathbf{a}, \mathbf{b} \times 10 ; \mathbf{c} \times 30)$

or white patch as the sensitive and specific patterns in this patient group.

In the current study including 69 patients with PCA, we evaluated the potential benefit of a handheld dermatoscope, which is more readily accessible to most dermatologists than a videodermoscope.

\section{Follicular Features}

All our patients displayed absence of follicular openings, which has been accepted as a pathognomonic feature to distinguish between cicatricial and noncicatricial alopecia $[2,3,8]$. Nevertheless, it is not always observed in the early phases of DLE $[3,15]$ or DCS $[6,16]$. Thus, it is suggested that diagnosis of PCAs can be established more precisely if this feature is accompanied with pink-white appearance $[3,15]$. 
We evaluated yellow dots under three groups: yellow dots that are millimetric sized points are most commonly reported in noncicatricial alopecias such as alopecia areata (AA) [11], but were also detected in DCS [3, 6, 7]. Yellow dots with "3D" structure appear as a characteristic finding of DCS [2, 3]. Similarly, this study revealed a higher prevalence of yellow dots in DCS (83.3\%) than the others, whereas yellow dots with "3D" structure were confined to DCS. Hence, we think that yellow dots are a highly suggestive, while yellow dots with "3D" structure are a specific marker for DCS, confirming previous observations $[2,3,6,7]$. Large keratotic yellow dots representing follicular keratotic plugs [2, 3, 13, 17] were noted only in DLE (42.9\%) in this study, providing a diagnostic finding for this disease.

Black dots have been considered as a specific feature of AA [18]. Yet, subsequent studies showed that they are also visible in other types of alopecia including PCAs [3, 6-9, 19]. They were most common in DCS (83.3\%), consistent with earlier data [2,3].

White dots present as small, regular "pinpoint" dots matching to eccrine sweat gland ducts, and follicular openings on healthy or alopecic scalps [20]. They may also appear as fibrotic white dots that are larger with blurred borders, distributed irregularly in folliculocentric PCAs [21], particularly LPP [2, 3, 13]. As none of them were more frequent in any particular type of PCA in this study, our data indicate that they do not constitute a helpful diagnostic finding.

Blue-grey dots, which correlate with melanophages in papillary dermis, are defined as either a "target" pattern, that was mainly observed in LPP, or a "speckled" pattern, mostly observed in DLE [13]. We detected only "target" pattern blue-grey dots exclusively in LPP (25.9\%), so we regard their presence as another clue for LPP.

Follicular red dots were reported in active DLE [22]. However, we did not observe them in any of our patients, as previously noted by Rakowska et al. [2].

Perifolicular erythema has been proposed as a direct marker of disease activity in FFA [4], yet they were not seen in the present study, confirming earlier data [5].

We documented absence of vellus hairs in some of our patients with LPP, FFA, and DLE. Lacarruba et al. [5] declared that absence of vellus hairs in the frontotemporal hairline is a diagnostic hallmark of FFA. However, our findings are not in line with this conclusion, as we detected vellus hairs in three of our patients $(42.9 \%)$ with FFA.

Short vellus hairs are noted in noncicatricial alopecias [15], and in early treated scarring alopecias [16]. The current study revealed a higher prevalence of them in FD (47.1\%) than the others. Thus, this finding can be a useful marker for FD.

Regrowing hairs should be differentiated from short vellus hairs by their darkly pigmented upright shafts with pointed ends [16]. They were observed in just two patients with FD, most probably representing the hair regrowth in the early stages of the disease.

Our results show that broken hairs do not constitute a helpful clue, since we documented them in almost all types of PCA.

Tufted hairs are reported most frequently in FD, though they were also found in LPP and DCS $[3,23]$. Consistent with these prior studies, we observed them in both FD and LPP, being markedly significant in the former $(82.4 \%$ versus $18.5 \%)$. We thus consider them to be a highly sensitive finding for FD.

Pili torti has been regarded as a typical feature for PCAs [8]. We detected it in all types of PCA, with the highest incidence in FFA (71.4\%).

\section{Follicular and Interfollicular Features}

Pink-white appearance was noted in every patient in the present study, in accordance with literature $[2,3,8,9]$.

Yellow structureless areas have been defined as one of the most characteristic features of DCS [2]. However, our data did not present evidence for this observation, as we noted this finding in only one patient with DCS.

Honeycomb hyperpigmentation pattern results from chronic sun exposure of the balding scalp mainly due to PCAs $[8,11]$, while 
brown scattered pattern displaying a "dirty" appearance occurs in active DLE lesions [2], FD [3], and LPP [3]. We observed both pigment patterns in almost all types of PCA, without a significant difference. Therefore, they seem to represent a nondiagnostic finding.

Crust formation was most frequently encountered in FD (70.6\%), as reported by Abedini et al. [3].

Interfollicular scaling was detected in all types of PCA except PB in this study. A recent study reported that it was a specific finding for PCAs [3]. Yet, this is contrary to earlier data $[8,15]$ which identified this feature in any kind of alopecia, inflammatory scalp diseases, and even healthy subjects. Nevertheless, perifollicular scaling, which is predominantly observed in active LPP, is accepted as a sensitive sign for PCAs $[2,3]$. Consistently with this finding, we documented it most frequently in LPP (81.5\%). Peripilar casts are a kind of perifollicular scaling distinguished by formation of freely movable white tubular structures around hair shafts $[2,15]$. We encountered them significantly in LPP, supporting an earlier investigation [2] that emphasizes them as a highly characteristic feature for LPP. Yellowish tubular scaling has been described as the presence of a band of yellowish scales surrounding hairs tufts in FD [2]. There was a strong predominance of them in FD $(70.6 \%)$ in this study as well.

Pustules are known as another characteristic feature of FD, yet also reported in DCS [3]. Our data confirmed that they are highly indicative of FD.

Simple, and twisted red vascular loops of the scalp are appreciated at 50-fold or higher magnifications by videodermoscopy [11]. They were seen as red dots at 30-fold magnification in the current study. Although their determination is especially valuable in inflammatory scalp disorders rather than alopecia, this study displayed a higher prevalence of red dots in FD than the others, in agreement with prior data [8].

Interestingly, our results did not support the previous conclusion that arborizing red lines are a highly representative feature for DLE $[2,3,10]$ because we documented them in all types of PCA.

\section{Trichoscopic Features Observed in LPP}

Our study revealed a higher prevalence of perifollicular scaling and peripilar casts in LPP, as compared with the other types. Nevertheless, "target" pattern blue-grey dots are thought to be a distinctive finding, since they were confined to LPP. Our results are mostly in line with literature $[2,3,13]$; however, tufted hairs were more frequent $(18.5 \%)$ than previously reported (7\%) [3].

\section{Trichoscopic Features Observed in FD}

Tufted hairs, crust formation, yellowish tubular scaling, pustules, and red dots were most frequently documented in FD, as already mentioned in several reports [2, 3]. Furthermore, a higher prevalence of short vellus hairs was noted in FD (47.1\%) than the others. Therefore, we consider this finding as another suggestive sign of FD.

\section{Trichoscopic Features Observed in FFA}

Our data support recent work [2] suggesting that perifolicular scaling is generally less frequent and milder in FFA than LPP. Yet, pili torti was noted more frequently $(71.4 \%)$ than previously reported (26.7\%) [5], revealing a helpful marker for FFA. Moreover, as a novel finding, our study indicated that vellus hairs can be visible in FFA, in contrast to a previous report [5].

\section{Trichoscopic Features Observed in DLE}

We observed large keratotic yellow dots only in patients with DLE, compatible with literature suggesting that it is a principal feature of DLE $[2,3,13,17]$. Notably, interfollicular scaling was most frequently associated with DLE (85.7\%), while perifollicular scaling was documented in less than half of the patients. Brown scattered pigmentation was seen in a third of the subjects. Surprisingly, arborizing red lines and follicular red dots were not representative patterns for DLE. 


\section{Trichoscopic Features Observed in DCS}

Yellow dots with "3D" structure were found in every patient with DCS, proving it to be a pathognomonic feature of DCS, as previously reported [2, 3]. Besides, yellow dots and black dots were found to be highly characteristic findings in the context of PCAs. Notably, broken hairs were detected most frequently in DCS. Therefore, in line with literature $[6,7]$, we believe that a trichoscopic pattern including yellow dots, black dots, and broken hairs may occur in DCS mimicking AA.

\section{Trichoscopic Features Observed in BP}

Our data disclosed loss of follicular ostia on a background of porcelain-white areas without any sign of inflammation, as already mentioned by Rakowska et al. [2].

\section{Limitations}

The limitation of this study is the small sample size in some PCA subgroups.

\section{CONCLUSIONS}

The results of this study demonstrate the utility of a polarized-light handheld dermatoscope in clinical diagnosis of PCAs, yielding data commensurate with videodermoscopy. All trichoscopic features could be appreciated at tenfold magnification, excluding red dots that could only be viewed at a minimum magnification of $30 \times$. We suggest that trichoscopy using a handheld dermatoscope is a rewarding supplementary practice in clinical diagnosis of PCAs. Finally, as a novel observation, absence of vellus hairs does not seem to be an identifying feature for FFA.

\section{ACKNOWLEDGEMENTS}

Funding. No funding or sponsorship was received for this study or publication of this article. Article processing charges were funded by the authors

Authorship. All named authors meet the International Committee of Medical Journal Editors (ICMJE) criteria for authorship for this article, take responsibility for the integrity of the work as a whole, and have given their approval for this version to be published.

Disclosures. Özlem Karadağ Köse and A. Tülin Güleç have nothing to disclose.

Compliance with Ethics Guidelines. The study was approved by Başkent University Instutional Review Board and Ethics Committee (Project no: K17/171), and the study was conducted in accordance with the principles of the Declaration of Helsinki of 1964 and its later amendments. All participants gave informed consent before study participation.

Data Availiability. The manuscript has now associated data or the data will not be deposited.

Open Access. This article is distributed under the terms of the Creative Commons Attribution-NonCommercial 4.0 International License (http://creativecommons.org/licenses/ by-nc/4.0/), which permits any noncommercial use, distribution, and reproduction in any medium, provided you give appropriate credit to the original author(s) and the source, provide a link to the Creative Commons license, and indicate if changes were made.

\section{REFERENCES}

1. Rongioletti F, Christina K. Cicatricial (scarring) alopecias: an overview of pathogenesis, classification, diagnosis, and treatment. Am J Clin Dermatol. 2012;12:247-60.

2. Rakowska A, Slowinska M, Kowalska-Oledzka E, et al. Trichoscopy of cicatricial alopecia. J Drugs Dermatol. 2012;11:753-8.

3. Abedini R, Kamyab Hesari K, Daneshpazhooh M, et al. Validity of trichoscopy in the diagnosis of 
primary cicatricial alopecias. Int $\mathrm{J}$ Dermatol. 2016;55:1106-14.

4. Toledo-Pastrana T, Hernandez MJG, Camacho Martinez FM. Perifollicular erythema as a trichoscopy sign of progression in frontal fibrosing alopecia. Int J Trichology. 2013;5:151-3.

5. Lacarrubba F, Micali G, Tosti A. Absence of vellus hair in the hairline: a videodermatoscopic feature of frontal fibrosing alopecia. $\mathrm{Br} \mathrm{J}$ Dermatol. 2013;169:473-4.

6. Tosti A, Torres F, Miteva M. Dermoscopy of early dissecting cellulitis of the scalp simulates alopecia areata. Actas Dermosifiliogr. 2013;104:92-3.

7. Segurado-Miravalles G, Camacho-Martinez F, AriasSantiago S, et al. Trichoscopy of dissecting cellulitis of the scalp: exclamation mark hairs and white dots as markers of disease chronicity. J Am Acad Dermatol. 2016;75:1267-8.

8. Karadă Köse Ö, Güleç AT. Clinical evaluation of alopecias using a handheld dermatoscope. J Am Acad Dermatol. 2012;67:206-14.

9. Park J, Kim JI, Kim HU, et al. Trichoscopic findings of hair loss in Koreans. Ann Dermatol. 2015;27:539-50.

10. Chiramel MJ, Sharma VK, Khandpur S, et al. Relevance of trichoscopy in the differential diagnosis of alopecia: a cross-sectional study from North India. Indian J Dermatol Venereol Leprol. 2016;82:651-8.

11. Ross EK, Vincenzi C, Tosti A. Videodermoscopy in the evaluation of hair and scalp disorders. J Am Acad Dermatol. 2006;55:799-806.

12. Ankad BS, Beergouder SL, Moodalgiri VM. Lichen planopilaris versus discoid lupus erythematosus: a trichoscopic perspective. Int $\mathrm{J}$ Trichology. 2013;5:204-7.

13. Duque Estrada B, Tamler C, Sodre CT, et al. Dermoscopy patterns of cicatricial alopecia resulting from discoid lupus erythematosus and lichen planopilaris. An Bras Dermatol. 2010;85:179-83.

14. Inui $\mathrm{S}$, Nakajima $\mathrm{T}$, Shono $\mathrm{F}$, et al. Dermoscopic findings in frontal fibrosing alopecia: report of four cases. Int J Dermatol. 2008;47:796-9.

15. Mubki T, Rudnicka L, Olszewska M, et al. Evaluation and diagnosis of the hair loss patient: part II. Trichoscopic and laboratory evaluations. J Am Acad Dermatol. 2014;71:431.e1-11.

16. Rakowska A, Olszewska M, Czuwara J, et al. Dissecting cellulitis. In: Rudnicka L, Olszewska M, Rakowska A, editors. Atlas of Trichoscopy. 1st ed. London: Springer; 2012. p. 331-7.

17. Lanuti E, Miteva M, Romanelli P, et al. Trichoscopy and histopathology of follicular keratotic plugs in scalp discoid lupus erythematosus. Int J Trichology. 2012;4:36-8.

18. Inui $S$, Nakajima $T$, Nakagawa $K$, et al. Clinical significance of dermoscopy in alopecia areata: analysis of 300 cases. Int J Dermatol. 2008;47:688-93.

19. Kowalska-Oledzka E, Slowinska M, Rakowska A, et al. 'Black dots' seen under trichoscopy are not specific for alopecia areata. Clin Exp Dermatol. 2012;37:615-9.

20. Abraham LS, Pineiro-Maceira J, Duque-Estrada B, et al. Pinpoint white dots in the scalp: dermoscopic and histopathological correlation. J Am Acad Dermatol. 2010;63:721-2.

21. Kossard S, Zagarella S. Spotted cicatricial alopecia in dark skin. A dermoscopic clue to fibrous tracts. Australas J Dermatol. 1993;34:49-51.

22. Tosti A, Torres F, Misciali C, et al. Follicular red dots. Arch Dermatol. 2009;145:1406-9.

23. Annessi G. Tufted folliculitis of the scalp: a distinctive clinicohistological variant of folliculitis decalvans. Br J Dermatol. 1998;138:799-805. 